\title{
Infecciones por Staphylococcus aureus meticilino resistente adquirido en la comunidad: experiencia de 10 años en un hospital pediátrico de Rosario, Argentina Community-acquired methicillin-resistant Staphylococcus aureus infections: 10-years' experience in a children's hospital in the city of Rosario, Argentina
}

\author{
Dra. Gabriela Ensinck ${ }^{a}$ Bioq. Adriana Ernsta ${ }^{a}$ Dr. Gustavo Lazarte ${ }^{a}$, \\ Dra. Antonela Romagnoli ${ }^{a}$ Dra. Yanina Sguassero ${ }^{b}$, Lic. Nanci Míguez ${ }^{a}$, \\ Dr. Santiago López Papuccia, Dra. Alicia Alettia , Dra. Ana Chiossonea, \\ Lic. Fernanda Pigozzia y Lic. Matías Pinottia \\ Colaboradora: Dra. Ana Cantador
}

\section{RESUMEN}

Introducción: Las infecciones por Staphylococcus aureus meticilino resistente adquirido en la comunidad (SAMR-ac) son motivo de consulta frecuente en pediatría. La mayoría se presentan como infecciones de piel y partes blandas; sin embargo, en la última década, se ha constatado un aumento de las infecciones invasivas.

Objetivos: El objetivo principal es describir las características clínico-epidemiológicas de las infecciones producidas por SAMR-ac. El objetivo secundario es comparar la prevalencia, presentación clínica y susceptibilidad antibiótica con un período previo de estudio (1/200412/2007).

Material y métodos: Estudio descriptivo, prospectivo, de tipo transversal. Criterios de inclusión: niños con diagnóstico de infección por SAMR-ac admitidos en el Hospital de Niños de Rosario, período de enero de 2008 a diciembre de 2014. Criterios de exclusión: internación reciente, antibiótico o cirugía previa, comorbilidades o inmunocompromiso.

a. Servicio de Infectología, Hospital de Niños Víctor J. Vilela de Rosario.

b. Centro Rosarino de Estudios Perinatales (CREP).

c. Instituto Rosarino de Idiomas. Rosario, Santa Fe.

Correspondencia: Dra. Gabriela Ensinck, ensinck@intramed.net.ar

Financiamiento:

Ninguno que declarar.

Conflicto de intereses:

Ninguno que declarar.

Recibido: 6-7-2017

Aceptado: 7-9-2017 http: / / dx.doi.org/10.5546/ aap.2018.119

Texto completo en inglés:

http:/ / dx.doi.org/10.5546/aap.2018.eng.119

Cómo citar: Ensinck G, Ernst A, Lazarte G, et al. Infecciones por Staphylococcus aureus meticilino resistente adquirido en la comunidad: experiencia de 10 años en un hospital pediátrico de Rosario, Argentina. Arch Argent Pediatr 2018;116(2):119-125.

\section{INTRODUCCIÓN}

A partir de la década de los noventa, se ha constatado un aumento de las infecciones por Staphylococcus aureus $(S A)$ de la comunidad (SA-ac) a nivel mundial, $\mathrm{y}$, sobre todo, este aumento ha sido a expensas del Staphylococcus aureus meticilino resistente adquirido en la comunidad (SAMR-ac), que, si bien, inicialmente, se manifestó a manera de brotes en comunidades cerradas, logró diseminarse y afectar a la población en general. ${ }^{1-3}$

En este escenario, la sigla "SAMRac" hace referencia a infecciones por $S A$ meticilino resistente que han sido adquiridas por personas de la comunidad sin factores de riesgo personales ni asociados. Las cepas de SAMR-ac son fenotípica y genotípicamente diferentes a las cepas relacionadas con infecciones por SAMR adquiridas en hospitales o asociadas al cuidado de la salud, por lo que se considera que han surgido de novo en el seno de la comunidad. Poseen un casete cromosómico 
estafilocócico denominado mec (staphylococcal cassette chromosome mec; SCC mec, por sus siglas en inglés) tipo IV o $\mathrm{V}$, acarrean el gen mec $A$, que les confiere resistencia a los antibióticos $\beta$-lactámicos, y pueden acarrear también resistencia acompañante a otros grupos de antibióticos. ${ }^{4,5}$ Los SAMR-ac presentan resistencia a meticilina, variable sensibilidad a clindamicina y baja resistencia a trimetoprima-sulfametoxazol. ${ }^{6,7}$

Cabe destacar que estas cepas poseen también genes de una exotoxina llamada Panton-Valentine leukocidin $(P V L)$, que ha sido relacionada con invasividad, necrosis y mal pronóstico. ${ }^{8,9}$

Actualmente, las infecciones por SAMR-ac son un motivo de consulta frecuente en pediatría y, en la mayoría de los casos, se presentan como infecciones de piel y partes blandas (IPPB). ${ }^{10}$ En la última década, las infecciones invasivas, como bacteriemia, osteoartritis y supuración pleuropulmonar, han aumentado en la población pediátrica. ${ }^{1,2,11}$ Otra característica de las infecciones por SAMR-ac es ser recurrentes y su capacidad de producir focos secundarios. ${ }^{12}$

En 2003, aparecen los primeros reportes de infecciones por SAMR-ac en Sudamérica ${ }^{13} y$, en 2004, Paganini y col., ${ }^{14}$ publican el primer reporte sobre este tipo de infecciones en niños en Argentina. Considerando su alta prevalencia, es indispensable conocer la situación epidemiológica local a fin de mejorar el criterio frente a la toma de conductas terapéuticas.

El objetivo principal de este estudio es describir las características clínicoepidemiológicas de las infecciones producidas por SAMR-ac. El objetivo secundario es comparar la prevalencia, presentación clínica y susceptibilidad antibiótica con un período previo de estudio (1/2004-12/2007).

\section{POBLACIÓN Y MÉTODOS}

Se realizó un estudio descriptivo, prospectivo, de tipo transversal en niños y adolescentes con diagnóstico documentado de infección por SAMR-ac evaluados en el Hospital de Niños Víctor J. Vilela de la ciudad de Rosario durante el período comprendido entre enero de 2008 y diciembre de 2014. Se comparó con un estudio previo realizado en nuestro Hospital de tipo prospectivo y descriptivo entre enero de 2004 y diciembre de 2007.

En ambos períodos, se utilizaron los mismos criterios diagnósticos clínicos, de inclusión y exclusión, como así también la misma metodología microbiológica.
Los criterios de exclusión fueron los siguientes: pacientes que, en los seis meses previos al ingreso al estudio, hubiesen permanecido internados (al menos, por 24 horas), visitado un centro para cuidados de salud o estado en contacto con personal de la salud; hubiesen recibido antibióticos o bien tuvieran el antecedente de aislamiento previo de un SAMR; hubiesen sido portadores de un catéter de larga permanencia o se hubiesen sometido a hemodiálisis o a un procedimiento quirúrgico. Se excluyeron, además, pacientes con comorbilidades al momento del ingreso, tales como inmunosupresión, diabetes, corticoterapia, etc. No se excluyeron pacientes por no contar con todos los datos de las variables analizadas, pero fue aclarado en cada una de ellas.

La infección se consideró adquirida en la comunidad cuando se obtuvo desarrollo de $S A$ en, al menos, un cultivo tomado de un paciente ambulatorio o dentro de las primeras 48 horas de internación.

En cuanto al diagnóstico microbiológico, la muestra para cultivo se obtuvo por punciónaspiración o drenaje de la lesión de piel y partes blandas o de sitios estériles. El $S A$ se aisló y tipificó mediante pruebas microbiológicas convencionales y automatizadas según protocolos de trabajo vigentes en el Laboratorio de Microbiología del Hospital.

La sensibilidad a meticilina se determinó por difusión con discos de cefoxitina de 30 microgramos $(\mu \mathrm{g})$. La detección de halos menores de 20 milímetros $(\mathrm{mm})$ se consideró como resistencia. También se determinó la resistencia a rifampicina $(5 \mu \mathrm{g})$, a gentamicina $(10 \mu \mathrm{g})$, a trimetoprima-sulfametoxazol $(25 \mu \mathrm{g})$, a eritromicina $(15 \mu \mathrm{g})$ y a clindamicina $(2 \mu \mathrm{g})$. Los antibiogramas se interpretaron según normas del Clinical \& Laboratory Standards Institute (CLSI, 2014).${ }^{1515}$ La resistencia inducible a clindamicina se identificó colocando el disco de esta droga a una distancia de $25 \mathrm{~mm}$ del disco de eritromicina en el antibiograma (D test). La concentración inhibitoria mínima (CIM) a la vancomicina se realizó por microdilución o el método de dilución en agar utilizando tiras E-test.

Se diseñó una ficha para la recolección de los siguientes datos: sexo, edad, manifestaciones clínicas, localización de las lesiones y sensibilidad antibiótica del microorganismo aislado.

El análisis de los datos se realizó de forma global. Se utilizó el programa SAS 9.2 para realizar los análisis estadísticos. Las variables 
categóricas se describieron como números y porcentajes. Se calculó, además, la razón de odds (RO) y el intervalo de confianza (IC) del 95\% para los resultados principales. Las variables continuas se describieron como mediana y rango. Las comparaciones entre los grupos del estudio se realizaron con la prueba $t$ de Student. El nivel de significación estadística utilizado fue $\alpha=0,05$.

El protocolo de investigación fue avalado por el Comité de Ética de la Secretaría de Salud Pública de la Municipalidad de Rosario y aprobado por el Comité de Docencia e Investigación del Hospital de Niños Víctor J. Vilela. Antes de la inclusión del niño en el estudio, un miembro del equipo de investigación informó a los padres o tutores sobre sus objetivos y el carácter descriptivo, y destacó que la participación no modificaría la conducta clínica ni la indicación de tratamiento del niño. El equipo de investigación garantizó el anonimato y la confidencialidad de los datos.

\section{RESULTADOS}

Durante el período de estudio (2004-2014), se identificaron 728 niños con diagnóstico de infección por $S A$, de los cuales $529(73 \%)$ tuvieron infecciones por SAMR-ac (Gráfico 1). En este escenario, se observó que la tasa de incidencia de las infecciones por SAMR-ac varió de 12,2/ 10000 egresos hospitalarios en el año 2004 ( $1^{\text {er }}$ año del $1^{\text {er }}$ período) a 145/10000 en 2014 (último año del $2^{\circ}$ período), o sea, un aumento de 12 veces entre los años extremos del período de estudio.

Las características clínicas y epidemiológicas fueron estudiadas en una muestra de 456 niños con 520 infecciones (un niño tuvo más de un

GRÁFICO 1. Número de pacientes con infecciones por Staphylococcus aureus meticilino resistente adquirido en la comunidad por año. Período 2004-2014. Hospital de Niños Víctor J. Vilela de Rosario

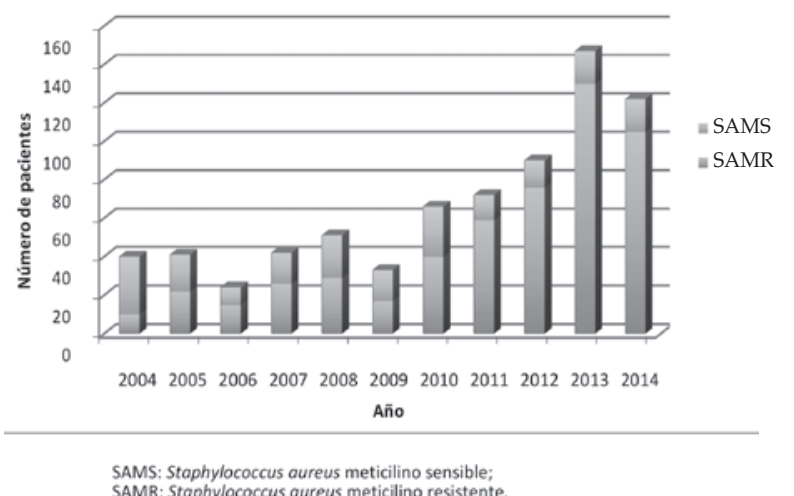

sitio de infección) por SAMR-ac evaluados en el período 2008-2014. El 57,6\% ( $\mathrm{n}=263)$ eran varones y la mediana de la edad fue 60 meses (1-202). Las características generales de la muestra del estudio se presentan en la Tabla 1.

De las 520 presentaciones clínicas de la muestra, las más frecuentes fueron IPPB $(75 \%$, $\mathrm{n}=391$ ), que incluyeron celulitis abscedadas, celulitis, impétigo y miositis; infecciones osteoarticulares $(8 \%, \mathrm{n}=43)$; infecciones pleuropulmonares $(6 \%, \mathrm{n}=30)$ y sepsis $(5 \%$, $\mathrm{n}=24)$. Cabe destacar que 40 niños $(8,7 \%)$ presentaron dos o más focos de infección. Se requirió drenaje quirúrgico como parte del tratamiento en 377 casos $(72,5 \%)$. Se registraron 125 infecciones invasivas (24\%) y dos muertes.

La localización de las infecciones se pudo estudiar en $392(86 \%)$ de los 456 niños, de los cuales, en $33(8 \%)$, hubo más de un sitio de infección. Las localizaciones más comunes fueron miembros inferiores y glúteo $(58 \%, \mathrm{n}=228)$, y les siguieron tórax $(16 \%, \mathrm{n}=62)$, miembros superiores $(11 \%, \mathrm{n}=45)$, cabeza y cuello $(11 \%, \mathrm{n}=42)$, abscesos profundos $(1 \%, \mathrm{n}=3)$ y bacteriemia $\sin$ foco $(0,5 \%, \mathrm{n}=2)$.

Se obtuvo aislamiento de SAMR-ac a partir del cultivo de 488 muestras correspondientes a IPPB $(80 \%, n=390)$, hemocultivos $(8 \%, n=37)$, líquido pleural $(5 \%, \mathrm{n}=25)$, líquido articular $(5 \%, \mathrm{n}=24)$, punción ósea $(1 \%, \mathrm{n}=5)$, líquido pericárdico $(0,43 \%, \mathrm{n}=2)$ y punción de dos abscesos profundos. En 25 niños (5\%), se obtuvo el SAMR-ac en más de un muestra (Tabla 2).

El patrón de sensibilidad antibiótica se realizó en los 488 aislamientos (hubo más de un aislamiento por paciente), y se observó resistencia exclusiva a oxacilina en 419 (86\%). Además, se constató resistencia acompañante a eritromicina en 44 cepas $(9 \%)$, a clindamicina en $34(7 \%)$ y a gentamicina en $29(6 \%)$. La combinación más frecuente fue la resistencia a eritromicina más clindamicina. No hubo resistencia a trimetoprimasulfametoxazol, rifampicina ni ciprofloxacina.

La sensibilidad a vancomicina se pudo estudiar en 376 aislamientos, de los cuales $359(95,47 \%)$ tuvieron una $\mathrm{CIM} \leq 1 \mathrm{ug} / \mathrm{ml}$ y 17 (4,53\%) cepas, una CIM de $1,5 \mathrm{ug} / \mathrm{ml}$. Ninguna cepa tuvo $\mathrm{CIM} \geq 2$.

En el segundo período, se constató un aumento de las infecciones ostearticulares y pleuropulmonares; sin embargo, no hubo un mayor riesgo de desarrollar infecciones invasivas respecto al primer período. Infecciones invasivas: $111 / 520$ vs. $15 / 78, \mathrm{OR}=1,1399$ (IC: $0,6250-2,0788$ ), $\mathrm{P}=0,6694$ (Tabla 3). 
Las diferencias en términos de resistencia antibiótica para gentamicina, clindamicina y eritromicina no fueron estadísticamente significativas entre ambos períodos (Tabla 4). No se registró resistencia a trimetoprimasulfametoxazol, rifampicina ni ciprofloxacina en ninguno de los dos períodos.

\section{DISCUSIÓN}

El aumento de las infecciones por SAMRac, principalmente, como IPPB, ha llevado a acrecentar tres o cuatro veces la consulta médica. ${ }^{16}$ Un extenso trabajo realizado en el año 2007 en hospitales pediátricos de nuestro país da cuenta del incremento de esta infección en

TABla 1. Características clínicas y epidemiológicas de los niños con infecciones por Staphylococcus aureus meticilino resistente adquirido en la comunidad $(n=456)$. Período 2008-2014. Hospital de Niños Víctor J. Vilela de Rosario

\begin{tabular}{|c|c|c|}
\hline Variables & Número & Porcentaje (\%) \\
\hline Varones & 263 & 57,6 \\
\hline \multicolumn{3}{|l|}{ Grupos etarios } \\
\hline$<12$ meses & 48 & 10,52 \\
\hline 12-60 meses & 253 & 55,48 \\
\hline$>60$ meses & 155 & 34 \\
\hline \multicolumn{3}{|l|}{ Manifestación clínica** } \\
\hline IPPB & 391 & 75 \\
\hline Infecciones pleuropulmonares & 30 & 6 \\
\hline Infecciones osteoarticulares & 43 & 8 \\
\hline Sepsis & 24 & 5 \\
\hline Otras & 46 & 6 \\
\hline \multicolumn{3}{|l|}{ Localización de las lesiones } \\
\hline M. inferiores y glúteos & 228 & 58 \\
\hline Tórax & 62 & 16 \\
\hline M. superiores & 45 & 11 \\
\hline Cabeza y cuello & 42 & 11 \\
\hline Otros & 15 & 4 \\
\hline Sensibilidad antibiótica & & 0 \\
\hline Oxacilina & 366 & 85 \\
\hline Eritromicina & 38 & 9 \\
\hline Clindamicina & 30 & 7 \\
\hline Gentamicina & 25 & 6 \\
\hline \multicolumn{3}{|l|}{ Aislamiento microbiológico } \\
\hline $\mathrm{IPPB}^{*}$ & 390 & 80 \\
\hline Hemocultivos & 37 & 8 \\
\hline Líquido pleural & 25 & 5 \\
\hline Líquido articular & 24 & 5 \\
\hline Punción ósea y abscesos prof. & 5 & 1 \\
\hline Líquido pericárdico & 2 & 0,4 \\
\hline
\end{tabular}

* Algunos niños tuvieron más de una forma de presentación clínica.

IPPB: infección de piel y partes blandas.

TABLA 2. Sitio de aislamiento de las infecciones por Staphylococcus aureus meticilino resistente adquirido en la comunidad. Período 2008-2014. Hospital de Niños Víctor J. Vilela de Rosario

\begin{tabular}{lcc} 
Aislamiento de SAMR-ac & Cantidad (488) & Porcentaje \\
\hline PPB & 390 & 80,00 \\
Hemocultivo & 37 & 8,11 \\
Líquido pleural & 25 & 5,48 \\
Líquido articular & 24 & 5,26 \\
Punción ósea & 5 & 1,10 \\
Punción de abscesos & 2 & 0,44 \\
Líquido pericárdico & 2 & 0,44 \\
Otros & 3 & 0,66 \\
Total & 488 & 100,00 \\
\hline
\end{tabular}

SAMR-ac: Staphylococcus aureus meticilino resistente adquirido en la comunidad; PPB: piel y partes blandas. 
internación y refiere un porcentaje de meticilino resistencia en las cepas estudiadas del $61 \% .{ }^{17}$ Otros importantes estudios realizados en Argentina entre los años 2012 y 2014 describen los clones circulantes en la población pediátrica: el más frecuente es el ST5-IV-PVL+ con un porcentaje de meticilino resistencia cercana al $55 \% \cdot{ }^{18,19}$ En nuestra experiencia de 10 años, se observó un incremento de, aproximadamente, 3 veces de las infecciones producidas por SA-ac, con un promedio de infecciones por SAMR-ac de $73 \%$, y se constató un incremento de la tasa de infecciones por SAMR-ac de 12 veces respecto a los egresos hospitalarios entre el año de inicio y el final del estudio (de 2004 a 2014).

El promedio de edad de estos pacientes fue cercano a 5 años; sin embargo, más de la mitad se ubicaron por debajo de los 4 años, datos similares a los observados en otras publicaciones nacionales. ${ }^{14,20,21}$ No hubo predominio de sexo, a diferencia de lo publicado en otras series, en las que se observó un claro predominio del sexo masculino y se argumentó como antecedente un mayor número de traumatismos en niños varones..$^{20}$
Las IPPB fueron la forma de presentación clínica más frecuente del total de las infecciones. Las celulitis abscedadas fueron la presentación que ocupó el primer lugar dentro de las IPPB y les siguieron en frecuencia las celulitis sin abscedar, el impétigo y la foliculitis y las miositis. La miositis y fascitis son una forma grave de infección que requiere, muchas veces, tratamientos invasivos. ${ }^{17,22}$ Los autores postulan que hay correlación de estas lesiones y un factor de virulencia del $S A$ conocido como $P V L$. Esta es una citotoxina que causa destrucción de leucocitos y necrosis de tejidos, lo que, sin duda, facilita a este microorganismo la capacidad de producir abscesos. Un metaanálisis publicado avala la asociación de $P V L$ y lesiones de piel y partes blandas, y aleja la asociación de $P V L$ con infecciones invasivas. ${ }^{23,24}$

La segunda forma de presentación clínica más frecuente fueron las infecciones ostearticulares, lo que coincidió con otros autores ${ }^{25} \mathrm{La}$ tromboflebitis, en general, formó parte del complejo miositis-artritis y osteomielitis. Las infecciones pleuropulmonares ocuparon el tercer lugar, principalmente, como neumonías

TABLA 3. Formas de presentación clínica de las infecciones por Staphylococcus aureus meticilino resistente adquirido en la comunidad. Período 2004-2007 y 2008-2014. Hospital de Niños Victor J. Vilela de Rosario

\begin{tabular}{|c|c|c|c|}
\hline \multirow{2}{*}{\multicolumn{2}{|c|}{$\begin{array}{c}\text { 2004-2007 } \\
\text { 78 infecciones / } 73 \text { pacientes } \\
59(75,6 \%)\end{array}$}} & \multicolumn{2}{|c|}{$\begin{array}{c}2008-2014 \\
520 \text { infecciones / } 456 \text { pacientes }\end{array}$} \\
\hline & & $391(75 \%)$ & NS (p: 0,6879) \\
\hline Osteoarticular & $4(5 \%)$ & $43(8 \%)$ & NS (p: 0,3413$)$ \\
\hline $\mathrm{SPP} / \mathrm{NN}$ & $1(1,2 \%)$ & $30(6 \%)$ & NS (p: 0,1299) \\
\hline Sepsis & $4(5,12 \%)$ & $24(4,61 \%)$ & NS (p: 0,8416$)$ \\
\hline Adenitis & $4(5,12 \%)$ & $18(3,46 \%)$ & NS (p: 0,4688$)$ \\
\hline Abscesos prof, & $2(2,5 \%)$ & $3(0,57 \%)$ & NS (p: 0,1090$)$ \\
\hline Tromboflebitis & $1(1,2 \%)$ & $3(0,57)$ & NS (p: 0,5071$)$ \\
\hline Pericarditis & $1(1,2 \%)$ & $3(0,57)$ & NS (p: 0,5071$)$ \\
\hline Endocarditis & $1(1,2 \%)$ & $2(0,38 \%)$ & NS (p: 0,3387 \\
\hline Peritonitis & $0(0 \%)$ & $2(0,38 \%)$ & NS \\
\hline Mediastinitis & $1(1,2 \%)$ & $1(0,2 \%)$ & NS (p: 0,1871) \\
\hline
\end{tabular}

IPPB: infecciones de piel y partes blandas; SPP/NN: supuraciones pleuropulmonares/neumonía nosocomial; NS: no significativo. Infecciones invasivas $111 / 520$ vs. 15/78, OR=1,1399 (IC: 0,6250-2,0788), $p=0,6694$.

TABLA 4. Resistencia acompañante a oxacilina en las infecciones por Staphylococcus aureus meticilino resistente adquirido en la comunidad. Período 2004-2007 y 2008-2014. Hospital de Niños Víctor J. Vilela de Rosario

Resistencia acompañante a oxacilina en ambos períodos

\begin{tabular}{|c|c|c|c|c|}
\hline Antibiograma & $2004-2007(78)^{*}$ & $2008-2014(488)^{*}$ & RO & \\
\hline Gentamicina & 3,2 & 5,6 & $\mathrm{RO}=1,97$ & $\mathrm{IC}=(0,46 ; 8,53)$ \\
\hline Clindamicina & 6,5 & 7,2 & $\mathrm{RO}=1,26$ & $\mathrm{IC}=(0,43 ; 3,68)$ \\
\hline Eritromicina & 6,5 & 9,3 & $\mathrm{RO}=1,66$ & $\mathrm{IC}=(0,58 ; 4,78)$ \\
\hline
\end{tabular}

* Microorganismos analizados.

RO: razón de odds; IC: intervalo de confianza. 
necrotizantes y supuraciones pleuropulmonares. Se describe, a nivel mundial, un aumento de los casos de neumonía por SAMR en niños, que se presentan, principalmente, como empiemas. ${ }^{26,28}$

En una experiencia realizada en nuestro Hospital, aún no publicada, las infecciones pleuropulmonares se presentaron en el 100\% de los casos como empiemas y 50\% de ellas, con necrosis y abscesos. El 50\% de los cuadros pleuropulmonares se acompañaron de sepsis. ${ }^{29}$ De los cuadros de sepsis, 2 casos se presentaron como sepsis sin foco con el desarrollo de lesiones en piel similares a meningococcemia y solo estos dos pacientes fallecieron durante el segundo período de estudio. Esta baja letalidad $<1 \%$ es similar a la publicada por otros autores. ${ }^{17,22} \mathrm{El}$ resto de las presentaciones, tales como adenitis, endocarditis, pericarditis, etc., fueron mucho menos frecuentes.

Los aislamientos fueron obtenidos de IPPB en 3/4 partes de los casos; $8 \%$ se aisló de hemocultivos, porcentaje similar a lo reportado por otros centros de nuestro país, ${ }^{21}$ seguidos por líquido pleural y articular.

El $80 \%$ de las cepas fueron solo resistentes a oxacilina, con una resistencia acompañante menor del $10 \%$ para eritromicina, clindamicina y gentamicina. No se constató resistencia a trimetoprima-sulfametoxazol, rifampicina ni ciprofloxacina. En cepas con resistencia a eritromicina, se estudió la resistencia inducible a clindamicina a través del D-test. Resistencias menores del $15 \%$ permiten a estos antibióticos formar parte del arsenal terapéutico de nuestro Hospital. ${ }^{30,31}$ La CIM a vancomicina fue $\leq 1$ en el 95\% de las cepas y ninguna cepa tuvo CIM $\geq 2$, dato de gran relevancia terapéutica, ya que la vancomicina es la droga de elección en el tratamiento de formas bacteriémicas y graves de esta infección en pediatría. ${ }^{32}$

El tratamiento quirúrgico fue requerido en $2 / 3$ partes de los pacientes. Sin duda, por la capacidad del microorganismo de producir abscesos y necrosis, el drenaje de las colecciones es una pieza fundamental en el tratamiento de estas infecciones. ${ }^{33}$

Comparando ambos períodos de estudio, se puede observar, en el segundo, un mayor número y variedad de infecciones invasivas, principalmente, ostearticulares y pulmonares; sin embargo, el riesgo de desarrollar infecciones invasivas entre ambos períodos no tuvo relevancia estadística. ${ }^{11}$

El patrón de resistencia antibiótica se mantuvo estable en ambos períodos. Los porcentajes de resistencia acompañante fueron similares a los publicados por otros autores de nuestro país. ${ }^{17,21}$

Dentro de las limitaciones de nuestro estudio, se puede mencionar que se realizó en un único centro y que, a pesar de ser un trabajo prospectivo, hubo datos que no se registraron en forma uniforme entre el primer y el segundo período. La tasa de IPPB puede tener la limitación de que no a todas las lesiones de piel se les realiza estudio microbiológico. El bajo número de fallecidos no permite evaluar el impacto de esta infección en la mortalidad. También se debe destacar que nuestros pacientes eran previamente sanos, sin antecedentes.

La fortaleza es, sin duda, el número de pacientes y el período de 10 años de estudio, lo que nos permitió evaluar el aumento en el número de infecciones, el incremento de la meticilino resistencia y la estabilidad en la resistencia antibiótica acompañante.

En conclusión, las infecciones por SA-ac se han incrementado en los últimos años, a expensas del SAMR-ac. Las IPPB, como celulitis abscedadas, fueron la forma de presentación clínica más frecuente. De las formas invasivas, predominaron las ostearticulares y pulmonares, $y$, si bien estas fueron más numerosas en el segundo período, no se constató un aumento de invasividad con respecto al primero. La resistencia antibiótica acompañante se mantuvo estable en el tiempo, con cifras de resistencia menores del $15 \%$, lo que permitió utilizar dichos antibióticos en los tratamientos empíricos iniciales de acuerdo con la forma de presentación clínica de la infección.

La rápida instauración del tratamiento médico adecuado y el drenaje quirúrgico en los casos indicados impactará, sin duda, en la morbimortalidad de estos pacientes.

\section{REFERENCIAS}

1. Buckingham SC, McDougal LK, Cathey LD, et al. Emergence of Community-Associated Methicillin-Resistant Staphylococcus aureus at a Memphis, Tennessee Children's Hospital. Pediatr Infect Dis J 2004;23(7):619-24.

2. MillerLG, KaplanSL.Staphylococcusaureus:ACommunity Pathogen. Infect Dis Clin North Am 2009;23(1):35-52.

3. KirklandEB,AdamsBB.Methicillin-resistantStaphylococcus aureus and athletes. J Am Acad Dermatol 2008;59(3):494-502.

4. Berglund C, Söderquist B. The origin of a methicillinresistant Staphylococcus aureus isolate at a neonatal ward in Sweden - Possible horizontal transfer of a staphylococcal cassette chromosome mec between methicillin-resistant Staphylococcus haemolyticus and Staphylococcus aureus. Clin Microbiol Infect 2008;14(11):1048-56.

5. International Working Group on the Classification of Staphylococcal Cassette Chromosome Elements (IWG- 
SCC). Classification of staphylococcal cassette chromosome mec (SCCmec): Guidelines for reporting novel SCCmec elements. Antimicrob Agents Chemother 2009;53(12):4961-7.

6. Paganini H, Della Latta M, Muller B, et al. Estudio multicéntrico sobre las infecciones pediátricas por Staphylococcus aureus meticilino-resistente provenientes de la comunidad en la Argentina. Arch Argent Pediatr 2008;106(5):397-403.

7. Frei CR, Miller ML, Lewis JS, et al. Trimethoprimsulfamethoxazole or clindamycin for communityassociated MRSA (CA-MRSA) skin infections. J Am Board Fam Med 2010;23(6):714-9.

8. Adler A, Temper V, Block CS, et al. Panton-Valentine leukocidin-producing Staphylococcus aureus. Emerg Infect Dis 2006;12(11):1789-90.

9. Morgan MS. Diagnosis and treatment of Panton-Valentine leukocidin (PVL)-associated staphylococcal pneumonia. Int J Antimicrob Agents 2007;30(4):289-96.

10. Comité Nacional de Infectología de la SAP. Infecciones de piel y partes blandas en pediatría: consenso sobre diagnóstico y tratamiento. Arch Argent Pediatr 2014;112(1):96-102.

11. Boucher H, Miller LG, Razonable RR. Serious Infections Caused by Methicillin $\square$ Resistant Staphylococcus aureus. Clin Infect Dis 2010;51(Suppl 2):S183-97.

12. Elston DM. Community-acquired methicillin-resistant Staphylococcus aureus. J Am Acad Dermatol 2007;56(1):116.

13. Galiana Villar A. Infección por Staphylococcus aureus meticilino resistente adquirido en la comunidad. Arch Pediatr Urug 2003;74(1):26-9.

14. PaganiniDH, Verdaguer V, Rodríguez AC, et al. Infecciones causadas por Staphylococcus aureus resistentes a la meticilina en niños provenientes de la comunidad en niños de la Argentina. Arch Argent Pediatr 2006;104(4):295-300.

15. Rapoport M. Novedades 2014. Clinical and Laboratory Standards Institute (CLSI). 2014:187-192. [Acceso: 13 de septiembrede2017].Disponibleen:http:/ / antimicrobianos. com.ar / ATB / wp-content / uploads / 2014/06/ NOVEDADES-CLSI-2014v21.pdf.

16. Wallin TR, Hern HG, Frazee BW. Community-Associated Methicillin-Resistant Staphylococcus aureus. Emerg Med Clin North Am 2008;26(2):431-55.

17. Paganini MH, Della L. P, Muller OB, et al. Infecciones por Staphylococcus aureus resistente a meticilina adquiridas en la comunidad en niños antes sanos y en niños relacionados al hospital en la Argentina. Rev Chil Infectol 2009;26(5):406-12.

18. Egea AL, Gagetti $P$, Lamberghini R, et al. New patterns of methicillin-resistant Staphylococcus aureus (MRSA) clones, community-associated MRSA genotypes behavelike healthcare-associated MRSA genotypes within hospitals, Argentina. Int J Med Microbiol 2014;304(8):1086-99.

19. Sola C, Paganini H, Egea AL, et al. Spread of epidemic MRSA-ST5-IV clone encoding PVL as a major cause of community onset staphylococcal infections in Argentinean children. PLoS One 2012;7(1):e30487.
20. Ves Losada JE, Graziano AP, De Abreu M, et al. Infecciones graves por Staphylococcus aureus: características clínicas, sensibilidad antibiótica y uso de antimicrobianos. Serie de casos. Arch Argent Pediatr 2014;112(4):e152-5.

21. Pérez G, Martiren S, Reijtman V, et al. Bacteriemia por Staphylococcus aureus adquirido en la comunidad en niños : estudio de cohorte 2010-2014. Arch Argent Pediatr 2016;114(6):508-513.

22. Kaplan SL. Community-Acquired Methicillin-Resistant Staphylococcus aureus Infections in Children. Semin Pediatr Infect Dis 2006;17(3):113-9.

23. Tristan A, Bes M, Meugnier $\mathrm{H}$, et al. Global distribution of Panton-Valentine leukocidin-positive methicillinresistant Staphylococcus aureus, 2006. Emerg Infect Dis 2007;13(4):594-600.

24. Rouzic N, Janvier F, Libert N, et al. Prompt and successful toxin-targeting treatment of three patients with necrotizing pneumonia due to Staphylococcus aureus strains carrying the panton-valentine leukocidin genes. J Clin Microbiol 2010;48(5):1952-5.

25. Kaplan SL. Osteomyelitis in children. Infect Dis Clin North Am 2005;19(4):787-97.

26. Sicot N, Khanafer N, Meyssonnier V, et al. Methicillin resistance is not a predictor of severity in communityacquired Staphylococcus aureus necrotizing pneumoniaresults of a prospective observational study. Clin Microbiol Infect 2012;19(3):e142-8.

27. Li HT, Zhang TT, Huang J, et al. Factors associated with the outcome of life-threatening necrotizing pneumonia due to community-acquired Staphylococcus aureus in adult and adolescent patients. Respiration 2011;81(6):448-60.

28. Carrillo-marquez MA, Hulten KG, Hammerman W, et al. Staphylococcus aureus Pneumonia in Children in the Era of community-acquired methicillin-resistence at Texas Children`s Hospital. Pedriatr infect Dis J 2011;30(7):545-50.

29. Ensinck G, Lazarte G, Ernst A, et al. Community-Acquired Methicillin Resistant Staphylococcus aureus (CA-MRSA) Pneumonia in a Children' s Hospital - Our experience. Poster presentado en $34^{\text {th }}$ Annual Meeting of thr European Society for Pediatric Infectious Diseases; 10-14 mayo 2016; Brighton, UK. ESP16-1086. [Acceso: 14 de septiembre de 2017]. Disponible en: https: / / www.collectlane.com/\# / post-event / 26/ posters / 13198 .

30. Chuck EA, Frazee BW, Lambert L, et al. The Benefit of Empiric Treatment for Methicillin-ResistantStaphylococcus Aureus. J Emerg Med 2010;38(5):567-71.

31. Messina AF, Namtu K, Guild M, et al. Trimethoprimsulfamethoxazole Therapy for Children With Acute Osteomyelitis. Pediatr Infect Dis J 2011;30(12):1019-21.

32. Hoang J, Dersch-Mills D, Bresee L, et al. Achieving Therapeutic Vancomycin Levels in Pediatric Patients. Can J Hosp Pharm 2014;67(6):416-22.

33. Fergie J, Purcell K. The Treatment of Community-Acquired Methicillin-Resistant Staphylococcus aureus Infections. Pediatr Infect Dis J 2008;27(1):67-8. 